\title{
PRIMARY MALIGNANT MELANOMA OF ARYEPIGLOTTIC FOLD
}

\author{
S. CHHABRA, R. SEN, P. PARMAR, P. GUPTA, A. SHARMA and H. YADAV \\ Department of Pathology, Pt.B.D.S. Post Graduate Institute of Medical Sciences, \\ Rohtak - 124001 (Haryana) India
}

DOI: http://dx.doi.org/10.4314/gmj.v49i4.10

Corresponding Author: Dr. Padam Parmar

Conflict Of Interest: None declared

\section{SUMMARY}

Background: Primary malignant melanoma rarely arises from noncutaneous tissues that contain melanocytes. Head and neck mucosal melanomas account for $0.7 \%$ to $3.8 \%$ of all melanomas. Here we report a case of primary malignant melanoma of aryepiglottic fold.

Result: Here we reported a case of primary malignant melanoma of aryepiglottic fold.

Conclusion: Primary aryepiglottic fold melanoma is an exceptionally rare neoplasm with early distant metastases and aggressive fatal course. Early diagnosis and proper line of treatment are crucial for survival. Physicians should keep a high index of suspicion for diagnosis of this rare tumor especially in old age with pigmented or non-pigmented lesion in larynx.

\section{INTRODUCTION}

Melanomas are mostly cutaneous in origin. Primary malignant melanoma occasionally arises from noncutaneous tissues that contain melanocytes, such as leptomeninges, uvea, and gastrointestinal, respiratory, and genitourinary tracts. Head and neck mucosal melanomas account for $0.7 \%$ to $3.8 \%$ of all melanomas. ${ }^{1}$ Primary malignant melanoma of the mucosa is more common than secondary metastatic disease and occurring more commonly in the nasal cavity and the maxilla.

However, melanoma metastatic to the mucosa of the head and neck region has been documented most commonly in larynx, tongue, and tonsil. ${ }^{2}$ Primary melanoma of the larynx and trachea are very rare among the group of non-cutaneous melanomas. In primary melanoma of the larynx, the least common site is the subglottic mucosa. ${ }^{3}$ Here we present a case of primary malignant melanoma of aryepiglottic fold.

\section{CASE REPORT}

A 70 year old woman, with no significant past medical history, presented to Otorhinolaryngology outpatient department of our hospital due to progressive change
Email:drpadamparmar@gmail.com

of voice (hoarseness) for the past four months. She had no history of dysphagia, odynophagia, and otalgia. There was no symptom of obstructive lung disease. On examination, direct and indirect laryngoscopy revealed a pigmented and ulcerated lesion involving the aryepiglottic folds. Computed tomography (CT) scan of the neck confirmed aryepiglottic lesion.

Biopsy of the ulcerated lesion revealed sheets of malignant melanin containing cells involving the overlying squamous mucosa and extending into the lamina propria. The nuclei were significantly pleomorphic with prominent nucleoli and mitotic figures. The immune profile of neoplastic cells was strongly positive for HMB-45, S-100, vimentin and negative for cytokeratin, synoptophysin and CD34. Extensive physical examination by a dermatologist and nuclear imaging including positron emission tomography (PET) scan failed to reveal a primary source of malignant melanoma. So, the patient was diagnosed as primary malignant melanoma of aryepiglottic mucosa.

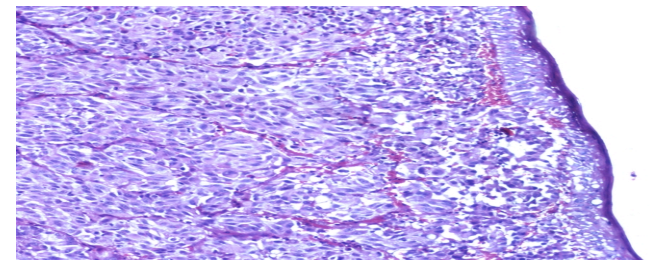

Figure 1 Photomicrograph showing origin of melanoma (H\&E, 100x)

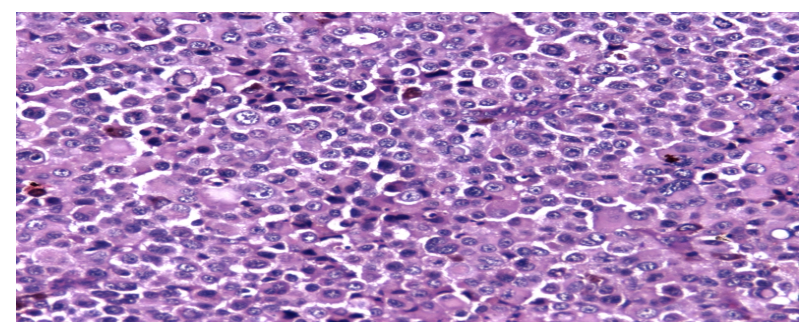

Figure 2 Photomicrograph showing sheets of melanoma cells with cytoplasmic pigment (H\&E, 400x) 


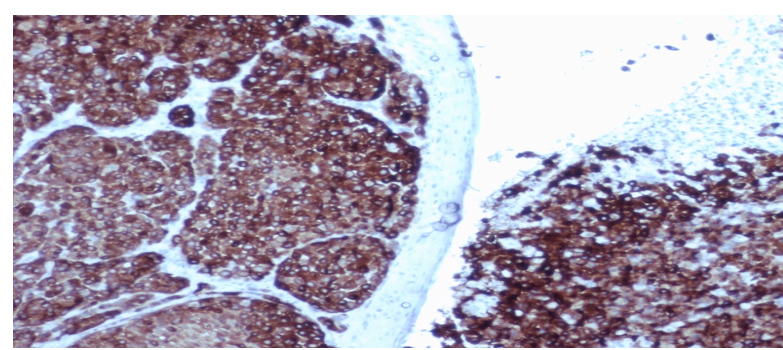

Figure 3 IHC showing positivity Melan A (for IHC, 200x)

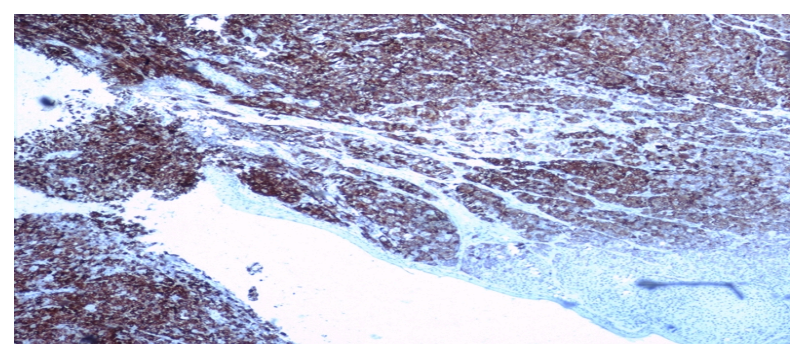

Figure 4 IHC showing positivity for HMB-45 (IHC, 200x)

\section{DISCUSSION}

Head and neck mucosal melanomas account for $0.7 \%$ to $3.8 \%$ of all melanomas. Majority of patients are white males in their sixth or seventh decade of life. Smoking is a major risk factor, but exposure to sunlight, human papilloma virus, chronic irritants, and carcinogenic compounds also play a role in pathogenesis of melanomas. Recently, several studies reported that malignant melanomas are result of an altered immune system, and many genes have been involved in its pathogenesis but this is not confirmed yet. ${ }^{1}$ Recent studies have shown an involvement of immune system dysregulation in mucosal melanomas with identification of certain genes like interleukin (IL17A) and CD70. This can be helpful in development of effective adjuvant immunotherapy for the treatment of melanomas. $^{1,3}$

Patients with primary malignant melanoma of the larynx usually present with complaints of hoarseness of voice and sore throat. If the size of tumor increases and obstructs the lumen of larynx, it can cause respiratory distress. Differentiation of primary from the secondary lesion may be challenging, especially as melanoma may sometime disappear from primary site after metastasis. On gross examination, cut surface of malignant melanoma may have slate gray, brown, or black pigmentation, which may be a clue to diagnosis. 1,3
Histopathological examination of tissue sections is needed for the diagnosis of melanoma. Hematoxylin and eosin staining typically shows pleomorphic, epithelioid, and/or spindle shaped malignant cells extending into adjacent lateral and overlying mucosa. Cells often contain dark brown cytoplasmic and nuclear melanin, however, some lesions are amelanotic. Presence of positivity of melanoma markers as S-100, HMB-45, Melan-A, and PNL-2 in melanoma cells through immunohistochemical staining is needed for confirmation of the diagnosis especially in amelanotic melanoma. Electron microscopy may identify the presence of melanosomes or premelanosomes. PET scan and/or magnetic resonance imaging $(\mathrm{MRI})$ can be used to stage primary melanoma. ${ }^{1,3}$

As compared to their cutaneous counterparts, mucosal melanomas are more aggressive and have worse prognosis with overall 5-year survival of less than $20 \%$. Poor prognosis is typically associated with early presentation of distant metastases. The treatment for mucosal melanomas of head and neck, including laryngeal lesions, is complete surgical excision, but sometimes it is difficult because of proximity of tumor to critical structures. Postoperative radiation therapy to the affected area has been effective in local spread of tumor in several retrospective series.

Gaissert et al performed a retrospective review of 360 primary tracheal tumors, in which only 1 patient was identified with tracheal melanoma. In a review of 259 cases of head and neck mucosal melanomas, the most common mucosal sites were the nasal cavity (69\%) and the oral cavity $(22 \%) .{ }^{4}$ Of the reported cases of primary laryngeal melanoma in the literature, Terada et al reviewed 38 patients, and found $80 \%$ were male, the mean age was 60 years, and the supraglottis was the most common primary site. The diagnosis of a head and neck mucosal melanoma is established through histopathological examination of biopsy. Criteria have been proposed for the differentiation of primary versus metastatic melanoma of the larynx and trachea. ${ }^{5}$ Durai et al reported a case of primary malignant melanoma of the epiglottis. ${ }^{6}$

Primary aryepiglottic fold melanoma is an exceptionally rare neoplasm with early distant metastases and aggressive fatal course. Early diagnosis and proper line of treatment are crucial for survival. Physicians should keep a high index of suspicion for diagnosis of this rare tumor especially in old age with pigmented or non pigmented lesion in larynx. 


\section{REFERENCE}

1. Ahmad S, Abdelghany M, Goldblatt C, Stark O, Masciotra N. A case of primary subglottic malignant melanoma with a successful surgical treatment. Case Rep Oncol Med. 2014;2014:968926.

2. Lanson BG, Sanfilippo N, Wang B, Grew D, DeLacure MD. Malignant melanoma metastatic to the larynx: treatment and functional outcome. Curr Oncol. 2010;17(4):127-32.

3. Mark J, Taliercio S, Karakla D. Primary laryngotracheal melanoma. Otolaryngol Head and Neck Surg. 2013;148:349-51.
4. Gaissert HA, Grillo HC, Shadmehr MB, Wright $\mathrm{CD}$, Gokhale M, Wain JC et al. Uncommon primary tracheal tumors. Ann Thorac Surg. 2006;82:268-72.

5. Terada T, Saeki N, Toh K, Uwa N, Sagawa $\mathrm{K}$, Mouri $\mathrm{T}$ et al. Primary malignant melanoma of the larynx: a case report and literature review. $A u$ ris Nasus Larynx. 2007;34:105-10.

6. Durai R, Hashmi S. Primary malignant melanoma of the epiglottis: a rare presentation. Ear Nose Throat J. 2006;85:274-7. 